\title{
A Recommendation Model for College Career Entrepreneurship Projects Based on Deep Learning
}

\author{
Yuan Feng ${ }^{1}$ and Weixian Huang $\mathbb{D}^{2}$ \\ ${ }^{1}$ Employment Guidance Teaching and Research Office, Yellow River Conservancy Technical Institute, Kaifeng, \\ Henan 475004, China \\ ${ }^{2}$ School of Entrepreneurship and Innovation, Shenzhen Polytechnic, Shenzhen Guangdong Province 518000, China
}

Correspondence should be addressed to Weixian Huang; cxcyhwx@szpt.edu.cn

Received 24 August 2021; Revised 25 September 2021; Accepted 1 October 2021; Published 21 October 2021

Academic Editor: Deepak Gupta

Copyright (C) 2021 Yuan Feng and Weixian Huang. This is an open access article distributed under the Creative Commons Attribution License, which permits unrestricted use, distribution, and reproduction in any medium, provided the original work is properly cited.

\begin{abstract}
The recommendation system is an active, personalized, and real-time technology platform proposed in the 1990s to solve the problem of information overload. The recommendation system can constantly adjust the recommendation results according to the real-time behaviors of users. In other words, if the user's interest changes, the recommendation system can present different information to the user. Therefore, the recommendation system is the best way to solve the problem of information overload in entrepreneurial projects. Based on the ConvMF algorithm, this paper proposes an entrepreneurial project recommendation algorithm based on a deep neural network and matrix decomposition. A deep neural network was established for the extraction of the hidden features of entrepreneurial projects, and a convolution neural network was used to process the text description information of entrepreneurial projects. One-hot coding was used to process the regional characteristics and financing round characteristics of entrepreneurial projects, and word embedding was used to process the label features of entrepreneurial projects. The implicit features of users are extracted from the user's rating matrix using matrix decomposition technology. Finally, recommendations are made according to the implicit characteristics of users and the items learned.
\end{abstract}

\section{Introduction}

Nowadays, China's universities have become the front line of innovation. The government and schools encourage college students to carry out innovative and entrepreneurial practice activities, apply teaching theoretical knowledge to extracurricular practice, and guide students to cultivate the ability of independent thinking and problem-solving, which improve the quality of personnel training and increase the employment rate. In the process of participating in innovation and entrepreneurship projects, students have accumulated a lot of knowledge, experience, and project data [1]. However, at present, the resource pool of innovation and entrepreneurship projects in colleges and universities is huge, the utilization rate of resources is low, and it is difficult to search for resources. We study the commonly used recommendation algorithms and build a personalized recommendation model suitable for college students' innovation and entrepreneurship resource library system, which can help users quickly find interesting resources [2].

The investigation of college students' entrepreneurship mainly investigates their entrepreneurial purposes and activities, while the GUESSS project is a global research, which has positive homework for understanding college students' entrepreneurship. The survey also covers a wide range, with about 200,000 students in 54 countries [3]. To study the factors of women's entrepreneurship development, we first list the important determinants and create a matrix to show the relationship at each level in the process, and we then quantify and define the above factors. Through this research method, it is helpful to encourage women to have entrepreneurial intentions and careers [4]. There are roughly three kinds of factors that affect women's entrepreneurship, namely, individual, environment, and, most importantly, politics. Nowadays, college graduates are more common, so it is particularly important to train and cultivate personal 
abilities and the motivation and ability to effectively participate in entrepreneurial activities during school. The entrepreneurial intention model for higher education came into being, which has a positive effect on improving students' entrepreneurial intention. The model is implemented as a practical digital application, mainly in three aspects: skill awareness, entrepreneurial resources, and network [5]. In view of the research of college students' entrepreneurial career and their adaptive behavior in occupations, this paper adopts the social cognitive model of career self-management [6]. We use 380 students who are about to graduate, based on the data, and use this model to verify and find the relationship between social cognitive career theory and adaptive exploration, which fills this unknown and has a certain influence on the education of career research and counseling in the future. It is helpful to study the time and type of individual entrepreneurship based on the opportunity to collect entrepreneurs' experiences. Using the data of Danish university graduates and employees, individuals are tracked. Compared with those who never started a business in terms of time, experience, and type of starting a business, early entrepreneurs earn higher wages and suffer fewer losses than late entrepreneurs [7]. This shows that the time type of entrepreneurial experience determines the subsequent career trajectory and the uncertainty of employers' value to it. Based on the data of college graduates and the theory of social cognitive career, this paper establishes a model of how different levels of contextual influence can help or inhibit the transformation of entrepreneurial intention into new entrepreneurial creation, which can evaluate the direct and larger background that affects the creation of new enterprises [8]. Entrepreneurship education for students is attracting attention from all walks of life, and it is also a factor affecting students' entrepreneurial intention, and it is also increasing significantly in schools around the world. Through the indepth study and review of relevant literature, as the basic background of knowledge, and then relying on narrative as a means of understanding and a form of communication, this research is positioned as a qualitative and explanatory phenomenological research [9]. With the advancement of education, rural students and young people are often not interested in engaging in agriculture as a career, MoMo, and prefer to work in cities. This paper studies this prominent phenomenon. Collecting 345 graduate students as samples, this paper analyzes the decisive factors of their ideas and intentions for agricultural entrepreneurship and shows that intention is the decisive factor, which is directly proportional to career planning and so on [10]. On the other hand, career planning, entrepreneurial ability, and agricultural family background (FB) can significantly predict agricultural entrepreneurial intention. This analysis has reference value for future teaching reform. Through investigation and study, college students' interest in entrepreneurship is closely related to their gender and race. This paper analyzes and compares their different attitudes and motives and then judges their interest points. For example, immigrant boys are much more enthusiastic about starting a business than local boys, girls are generally lower than boys, and boys will be greatly influenced by external factors, such as family, friends, and economic interests; girls are more motivated to use "internal factors" such as their skills and abilities. Entrepreneurship is equally important for both men and women to be flexible and independent [11]. College students are an important and valuable human force in a country. What is often discussed in society is the employability of graduates and whether they are willing to start entrepreneurial activities. The professional willingness of social entrepreneurs should be spread and cultivated during their student years, and entrepreneurship can be used as the starting point of their career, which requires college students to have the opportunity to get in touch with the concepts and opportunities of social entrepreneurship [12].

\section{Recommendation Process}

This paper designs a recommendation algorithm for entrepreneurial projects based on a deep neural network and matrix decomposition. This algorithm integrates the content information of entrepreneurial projects into the recommendation algorithm. Figure 1 shows the overall flow chart of the algorithm shown in this chapter. As can be seen from the figure, the recommendation flow mainly includes the following parts:

(1) Data Processing. It mainly includes the following: (i) scoring, calculating the score data of the user's entrepreneurial project according to the historical behavior data of the user's entrepreneurial project; (ii) sampling negative samples to generate negative samples based on the historical behavior data of users; and (iii) generation of the scoring matrix.

(2) Recommendation Model Construction. The construction of the recommendation model is the construction of the entrepreneurial project recommendation algorithm based on a deep neural network and matrix decomposition.

(3) Recommendation Result Generation. Use the trained model to generate recommendation results for users.

\section{Data Processing}

3.1. Processing of Implicit Feedback Data. In the research of personalized recommendation, users' interest in items is usually reflected by user rating data. For example, the wellknown MovieLens dataset and Netflix Prize dataset have clear user movie ratings [13]. However, in many practical application scenarios, there is often no clear user rating data, such as ordinary e-commerce websites such as JD.com and Taobao and news websites such as Today's Headlines and Baidu News. In this case, the user's implicit feedback data is usually used to measure the user's interest in the project [14]. User action data in the entrepreneurial project recommendation system is implicit feedback data. Next, the processing method of implicit feedback data is introduced.

Generally speaking, implicit feedback data is extracted from the interaction history between users and websites, for example, user clicks, browses, collections, and interaction time. For example, on e-commerce websites, users' reading, 

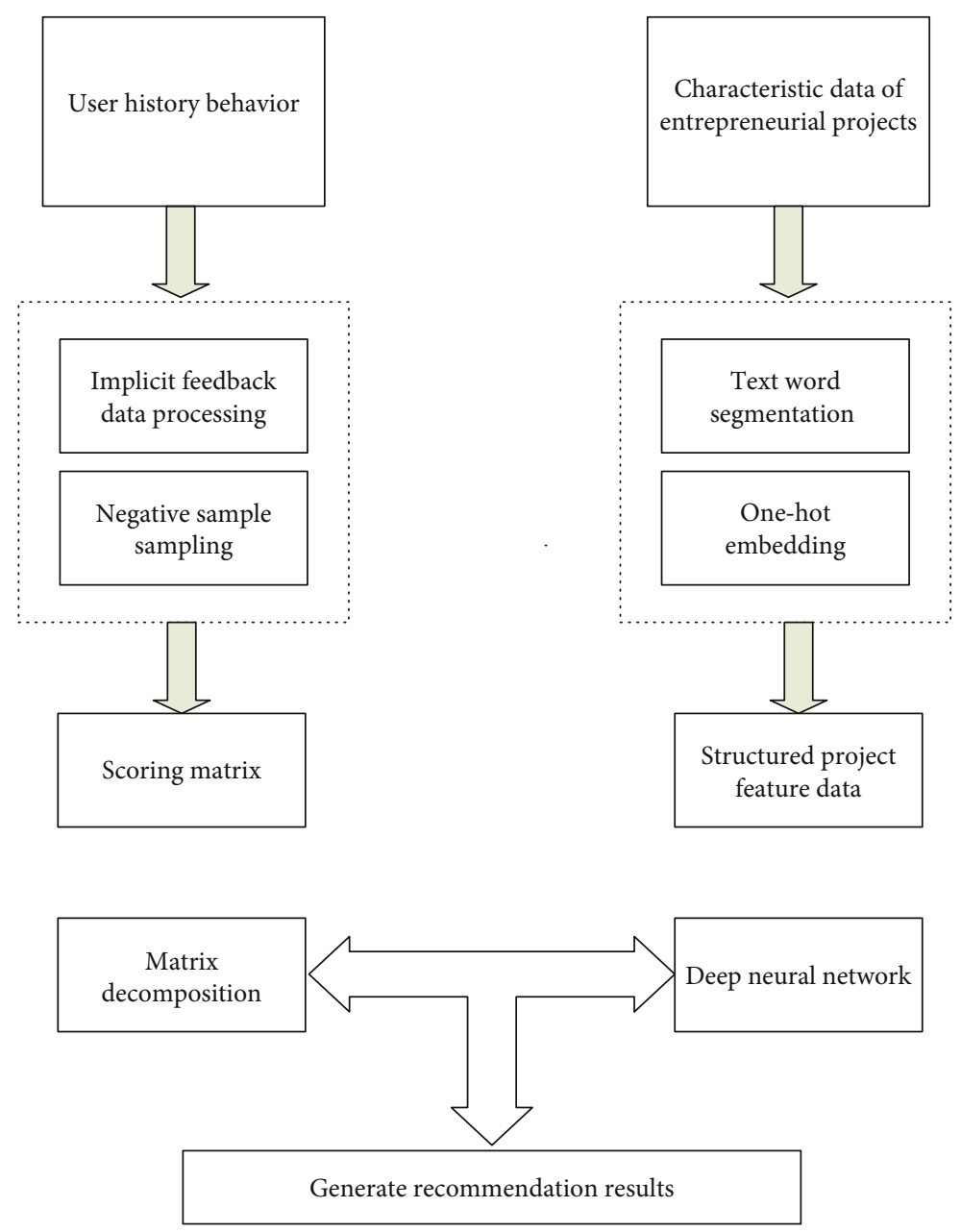

Figure 1: Recommendation flow chart.

TABLE 1: User interaction record table.

\begin{tabular}{lcccc}
\hline Users & Project 1 & Project 2 & $\ldots \ldots$ & Project $n$ \\
\hline Browse & $I_{11}$ & $I_{12}$ & $\ldots \ldots$ & $I_{1 n}$ \\
Attention & $I_{21}$ & $I_{22}$ & $\ldots \ldots$ & $I_{2 n}$ \\
Comments & $I_{31}$ & $I_{32}$ & $\ldots \ldots$ & $I_{3 n}$ \\
\hline
\end{tabular}

clicking, shopping cart addition, collection, purchase, and other behaviors reflect users' interest in goods [15].

In view of the research problem of this paper, the personalized recommendation system for entrepreneurial projects also has no specific user rating information. For users, the interaction history can be represented by Table 1: each row represents an action type (including reading, paying attention, and commenting, which are represented by 1, 2, and 3 , respectively), each column represents an entrepreneurial project, and $I_{i j}$ represents the number of rows $i$ of users on entrepreneurial projects $j$.

However, this raw interaction history cannot be used directly and needs to be processed before it can be used. The data type in Table 1 is the number of times, which refers to the number of times users browse, pay attention to, and comment on items, which is implicit feedback data. On the other hand, it can analyze the attention degree of users to the project, which reflects the personalized preference degree.

Users' interest in entrepreneurial projects is mainly reflected in two dimensions: the number of interactions and the types of interactive behaviors. First of all, in terms of the number of interactions, the more interactions the user has with the startup project, the more interest the user has in the project. For example, if the user browses item A five times and item $\mathrm{B}$ once, it indicates that the user is more interested in item A. Second, different interactive behaviors reflect different interests of users. For example, if a user comments on Project A and clicks Project B, it indicates that the user is more interested in Project A than Project B. Using the historical behavior of users, you can get the user score of starting the project. See Equation (1) for the calculation method:

$$
r_{w i}=\sum_{j=1}^{k} a_{i} q_{n i, j}
$$

where $a_{i}$ denotes the number of comments made by user $a$, $q_{n i, j}$ denotes the degree of interest in comments, and the 


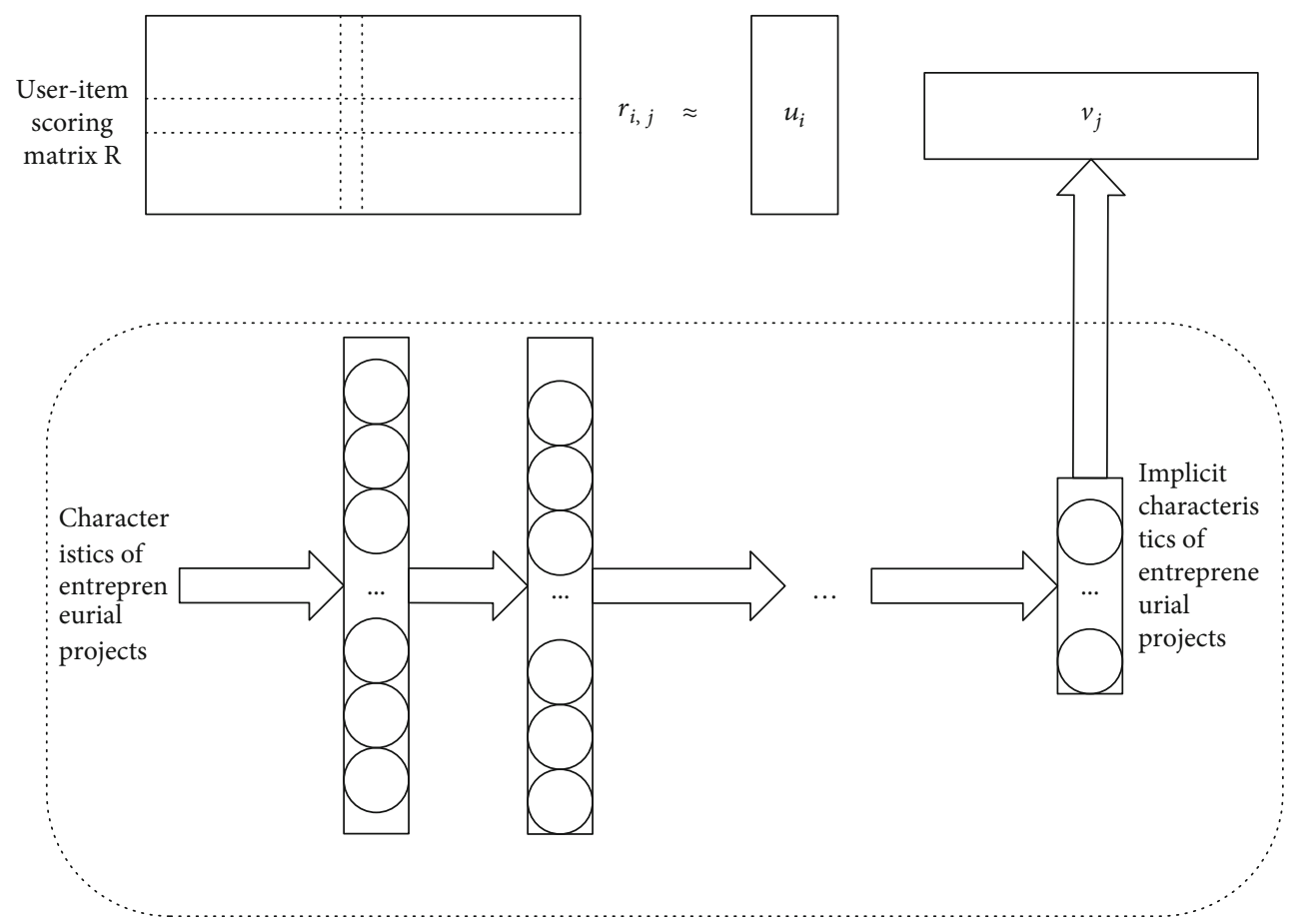

FIgURE 2: Structure diagram of the entrepreneurial project recommendation algorithm based on a matrix decomposition and deep neural network.

product of the different comment times and interest degree is the degree of interest in items in comments.

3.2. Negative Sampling. In a score-based recommendation system, users show their goodwill towards an item by scoring. For example, MovieLens and Netflix Prize datasets have user rating data on movies. If a user gives a movie a score of 5 , it means that the user likes the movie. If the user does not like a movie, the user will give the movie a very low score. Therefore, the score-based dataset contains both positive samples (items that the user likes) and negative samples (items that the user does not like) [16]. For the recommendation system of entrepreneurial projects, the user's action data is implicit feedback data. One of the characteristics of internal hidden feedback data is that there are only positive samples and no negative samples [17].

For the sampling of negative samples, Xiang Liang put forward the following sampling scheme in the book Practice of Recommendation System and achieved good results:

(1) For each user, it is necessary to ensure the balance of positive and negative samples

(2) For the negative sampling of users, select those elements that are very popular but have no user behavior

Generally speaking, websites will have columns similar to popular items, so popular items are easier for users to find. If the project is very popular and the user has no behavior, it can better indicate that the user is not interested in the project. For unpopular items, the exposure on the website may not be high, and users may not find them at all during browsing the website, so it is impossible to judge whether users are interested in the item. Therefore, aiming at the research problem [18], this paper adopts the sampling method proposed by Xiang Liang to carry out the negative sampling.

\section{Recommendation Algorithm Description}

As shown in Figure 2, the business project recommendation algorithm based on a deep neural network is constructed and decomposed into two parts: the business project recommendation algorithm based on a deep neural network and matrix decomposition. As an input element of a business project, the output is used to extract the implicit features of the project.

4.1. Deep Neural Network and Probability Matrix Decomposition Algorithm. The probability matrix factorization (PMF) algorithm is a matrix factorization algorithm [19]. The purpose of probability matrix factorization is to decompose the hidden characteristics of elements and users from the score matrix [20] and then introduce the probability matrix factorization algorithm used in this Table 1.

Assuming that there are $N$ users and $M$ items, the scoring matrix $R=R^{N \times M}$ can be obtained. The goal of matrix decomposition is to calculate the hidden features of users and items $U=R^{K \times N}$ and $V=R^{K \times M}$ by using the scoring matrix $R$. With the hidden features $U$ and $V$ of users and items, on the contrary, the unknown scores in the evaluation matrix $R$ are calculated by $U^{T} V$.

Firstly, the probability matrix decomposition algorithm assumes that the hidden feature vectors of users and items follow Gaussian distribution, and its expressions are shown in 


$$
\begin{aligned}
& P\left(U \mid \sigma_{U}^{2}\right)=\prod_{i=1}^{N} N\left(u_{i} \mid 0, \sigma_{U}^{2} I\right), \\
& P\left(V \mid \sigma_{V}^{2}\right)=\prod_{j=1}^{N} N\left(V_{i} \mid 0, \sigma_{v}^{2} I\right),
\end{aligned}
$$

where $N\left(x \mid \mu, \sigma^{2}\right)$ represents the probability density function of Gaussian distribution, $\mu$ represents the mean value, $\sigma^{2}$ represents the variance, $U$ and $V$ represent the implicit feature evidence of users and items, $U_{i}$ and $V_{j}$ represent the implicit feature vectors of users and items, and $I$ represents the unit matrix. $U$ and $V$ are obtained by matrix decomposition, which can better calculate the hidden characteristics of users and projects. $P$ represents the probability density function of Gaussian distribution.

In addition, it is assumed that the conditional probability of observation score data also follows Gaussian prior distribution, and the expression is shown in

$$
P\left(R \mid U, V, \sigma_{R}^{2}\right)=\prod_{i=1}^{n} \prod_{j=1}^{m}\left[N\left(R_{i, j} \mid U_{i}^{T}, V_{j} \sigma_{R}^{2}\right)\right]^{l_{i, i}^{n}} .
$$

The Bayesian formula can be used to obtain the postinspection probability of users and projects with hidden characteristics, as shown in

$P\left(U, V \mid R, \sigma_{R}^{2}, \sigma_{U}^{2}, \sigma_{V}^{2}\right) \propto P\left(R \mid U, V, \sigma_{R}^{2}\right) P\left(U \mid \sigma_{U}^{2}\right) P\left(V \mid \sigma_{V}^{2}\right)$.

Assuming that $W$ is the parameter set of the deep neural network and follows Gaussian distribution and that $X_{j}$ is the characteristic input of term $j$, the calculation method of term $j, V_{j}$, is shown in

$$
V_{j}=\operatorname{dnn}\left(W, X_{j}\right)+\epsilon_{j}
$$

In addition, $\operatorname{dnn}(\cdot)$ denotes the deep neural network, and $\epsilon_{j}$ denotes the random error and expresses the equation shown in Equation (7) according to Gaussian distribution:

$$
\epsilon_{j} \sim N\left(O, \sigma_{V}^{2}\right) I
$$

Assuming that the parameter set $W$ also obeys Gaussian distribution, the probability distribution of $W$ is shown in

$$
P\left(W \mid \sigma_{W}^{2}\right)=\prod_{k=1}^{p} N\left(W_{k} \mid 0, \sigma_{W}^{2}\right) .
$$

The conditional probability distribution of the implicit feature $V$ of the item is shown in

$$
P\left(V \mid W, X, \sigma_{V}^{2}\right)=\prod N\left(V_{j} \mid \operatorname{dnn}\left(W, X_{j}\right), \sigma_{V}^{2} I\right) .
$$

The probability distribution can be calculated by the Bayesian formula, as shown in

$$
\begin{aligned}
& P\left(U, V, W \mid R, X, \sigma^{2}, \sigma_{U}^{2}, \sigma_{v}^{2}, \sigma_{w}^{2}\right) \\
& \quad=P\left(R \mid U, V, \sigma^{2}\right) P\left(U \mid \sigma_{v}^{2}\right) P(V \mid R, W, X) P\left(W \mid \sigma_{w}^{2}\right) .
\end{aligned}
$$

4.2. Extraction of Implicit Features of Entrepreneurial Projects. Entrepreneurial projects contain a lot of specific information, especially the description of commercial projects, brand names and signature characteristics of commercial projects, regional characteristics of commercial projects, and advantages of commercial projects, including structured data of project content such as regions, domains and labels, and structured data such as project description texts. When using the traditional word-back model to simulate entrepreneurial projects, there are certain boundaries. On the other hand, the word-back model has many limitations, such as not finding the context information of text data and extracting very little feature data. On the other hand, using the same method to process all feature data indiscriminately will also affect the recommendation results.

In order to extract more effective features from these content data, this paper combines the convolution neural network, word embedding technology, and one-hot technology to establish a deep neural network to extract hidden features of entrepreneurial projects. The convolution neural network is used to deal with the text description of entrepreneurial projects, word embedding technology is used to deal with the label data of entrepreneurial projects, and one-hot is used to deal with the regions and regional characteristics of entrepreneurial projects. Figure 3 is the structure diagram of the deep neural network established in this paper. Next, the network structure will be described in detail.

In Figure 3, the text input, region, domain, and label are of different data types or have different attribute requirements. Different inputs are the result of processing for the performance of attribute characteristics. Different input attributes in Figure 3 need to process different attribute values. After processing, all attributes will be converted into a unified processing attribute format. The input data of different attribute formats are unified into a unified data type after different processing, which is convenient for the application of the deep learning model.

4.2.1. Input Layer. The input layer inputs the characteristic data of the collated project, including label data input, text data input, company field data input, and regional data input (four parts). The input data is as follows.

Step 1 (item label data input). The number of item labels is between 3 and 10 .

Step 2 (text description data entry for business projects). Text description data must separate words and enter them into the network in word order.

Step 3 (regional data input). Regional data input is a single discrete value, such as Beijing and Shanghai. 


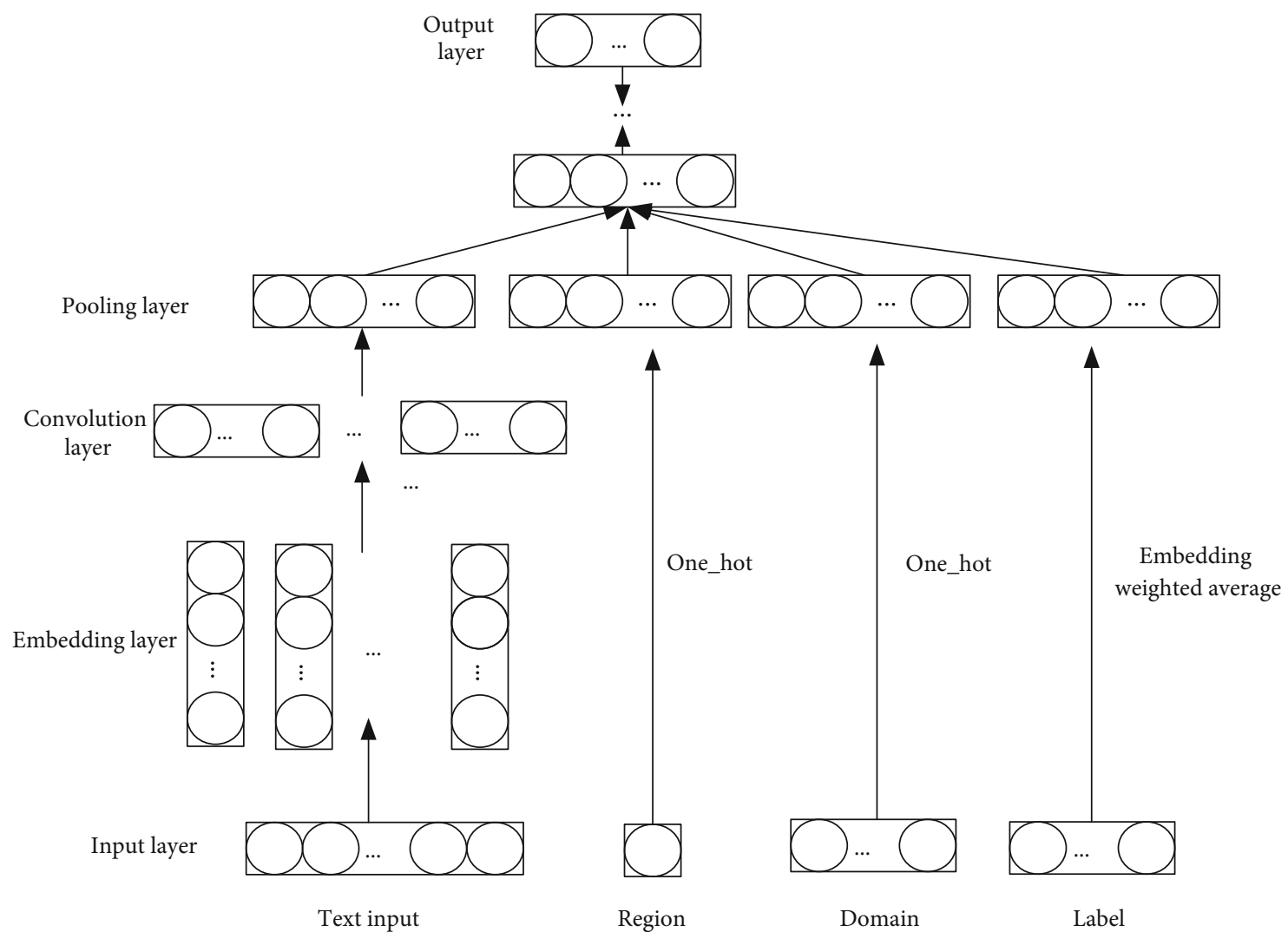

FIgURE 3: Deep neural network structure for extracting project features.

TABLE 2: The 10 tags most similar to the label "finance.".

\begin{tabular}{lc}
\hline Label & Similarity \\
\hline Insurance business & 0.8180 \\
Wealth management & 0.8172 \\
Nonperforming assets & 0.8161 \\
Fund & 0.8140 \\
Banking services & 0.8082 \\
Quantitative investment & 0.8064 \\
Asset securitization & 0.8047 \\
Foreign exchange & 0.8039 \\
Bill financing & 0.8027 \\
Microfinance & 0.8008 \\
\hline
\end{tabular}

Step 4 (domain data input). The domain information of the company is a sequence value, which can contain one or two values.

4.2.2. Label Data Processing of Entrepreneurial Projects. Each label is represented by a $k$-dimensional vector. The principle of the same vector is the same. The more similar the labels are, the closer the spatial distance is. Table 2 shows the 10 labels closest to "finance" and their similarity. In this paper, cosine similarity is used to calculate the similarity between different tags.

Because each item has multiple tags, the trained tag vector cannot be directly used as the input of the neural net-
TABLE 3: Content information of an item.

\begin{tabular}{lc}
\hline $\begin{array}{l}\text { Item number } \\
\text { Project name } \\
\text { Project area }\end{array}$ & 50000 \\
Project brief & $\begin{array}{c}\text { Business Information Technology } \\
\text { Entroduction services }\end{array}$ \\
introduction Information Technology is & $\begin{array}{c}\text { Business } \\
\text { the catering and entertainment industry }\end{array}$ \\
Item label & $\begin{array}{c}\text { Enterprise services, SAAS, APP, platform, } \\
\text { solutions, performance management }\end{array}$ \\
\hline
\end{tabular}

work. Assuming that the item has a tag set, then using the method $T=\left\{t_{1}, t_{2}, \cdots, t_{l}\right\}$ of adding and averaging tag vectors, where $t_{i} \in p^{l \times k}$, then the processed $p$ of the tag for each item can be expressed as

$$
p=\frac{1}{l} \sum_{i=1}^{I} t_{i}
$$

where $l$ represents the number of tags for the project and $p$ represents the processing result in Table 3.

Finally, $p$ is input into the deep neural network as the feature of item tag information. Table 4 shows the content information of the item with item number 50000, and the tag vector display of the item can be calculated using calculation formula (15). Table 4 shows the 10 tags closest to the item tag vector. 
TABle 4: Labels similar to Project 50000.

\begin{tabular}{lc}
\hline Label & Similarity \\
\hline Performance management & 0.9346 \\
IT operation and maintenance & 0.9298 \\
PAAS & 0.9167 \\
Enterprise mobility & 0.9088 \\
CMS & 0.9080 \\
Call center & 0.8976 \\
Mobile device management & 0.8972 \\
Server & 0.8901 \\
Customer service & 0.8731 \\
ERP & 0.8706 \\
\hline
\end{tabular}

4.2.3. The Processing Method of Text Description Data of Entrepreneurial Projects. In order to improve the accuracy of feature extraction of entrepreneurial projects, this paper uses a convolution neural network to process the file description information of entrepreneurial projects. The structure of the neural network containing text is shown in Figure 4. Next, the working mode of the text convolution neural network will be introduced in detail.

(1) Embedding Layer. An important concept of the embedding layer is embedded language, that is, distributed representation. The purpose is to use dense vectors to represent words to avoid the data sparsity problem of one-hot.

The purpose of the embedding layer is to transform the sequence of word segmentation results of text into the matrix and input it into the convolution neural network. The input of the embedding layer is in the order shown in

$$
D_{j}=\left(w_{1}, w_{1}, \cdots, w_{n j}\right) .
$$

For example, "Today's weather is fine" after the word segmentation order is as follows: "Today's weather is fine"; this word order can be used as the input of the embedding layer.

In the embedding layer, each word is represented by a $k$-dimensional vector (for example, 100 dimensions). After each word is represented by a $k$-dimensional vector, the results are spliced together, and each text sequence input can be converted into a matrix $D \in R^{N \times K}$ (where $N$ represents the length of the sequence and $K$ represents the dimension of the word vector), which can be expressed by

$$
D_{n \times k}=\left[\begin{array}{c}
-w_{1}- \\
-w_{2}- \\
\cdots \ldots \\
-w_{n-1}- \\
-w_{n}-
\end{array}\right],
$$

where $k$ represents the dimension of the word vector, $n$ represents the number of words in the text, and $w_{i}$ represents the word vector.

After the input text sequence is converted into the matrix, it can be used as the folding neural network input of text, in addition, how to quantify the words. In other words, there are two main ways to convert a word into a dense high-order vector:

(a) Pretrained word vectors can quantitatively express the relationship between words, such as the similarity between words. Vectorization of words is also beneficial to the modeling of natural language processing problems and reduces the sparsity of data. Therefore, word vectorization is a research hotspot in recent years. In recent years, many algorithms represented by word vectors have been proposed, such as Word2Vec, FastText, and WordRank. Here, the Word2Vec algorithm is the most widely used, which was proposed by Mikolov and others. The algorithm includes the CBOW language model and Skip-Gram language model. Because this part is not the main content of this paper, it will not be explained here. The word vector training tool in this paper is Word2Vec

(b) At the beginning of network training, the word vector is initialized randomly, and the word vector is always optimized and adjusted during training

(2) Convolution Layer. The main purpose of the convolution layer is to extract local features. Figure 5 folds a schematic diagram of the local field of view of a neural network with an input of the $8 \times 8$ matrix, with a local perceptual range of $2 \times 2$. All inputs in each local perceptual field are connected to the same hidden neuron, and $K$ and $L$ hide the original output, as shown in

$$
f\left(b+\sum_{i=0}^{n} \sum_{j=0}^{m} w_{i, j} a_{i+k l+j}\right) .
$$

The convolution kernel of the text convolution neural network is $P \times K$, the number of rows per convolution of $P$ is variable, and $K$ represents the dimension of the word vector, which is constant.

Therefore, the convolution operation of the text convolution neural network can be expressed by

$$
c_{i}^{j}=f\left(b_{c}^{j}+\sum_{i=0}^{p} \sum_{j=0}^{m} w_{i, j} D_{i+k, d+j}\right)
$$

where $c_{i}^{j}$ represents the activation value on a convolution kernel, $b_{c}^{j}$ is the offset term, $w_{i}$ and $j$ are the shared weights, $f$ is the activation function, and $D$ represents the input of the convolution layer. 


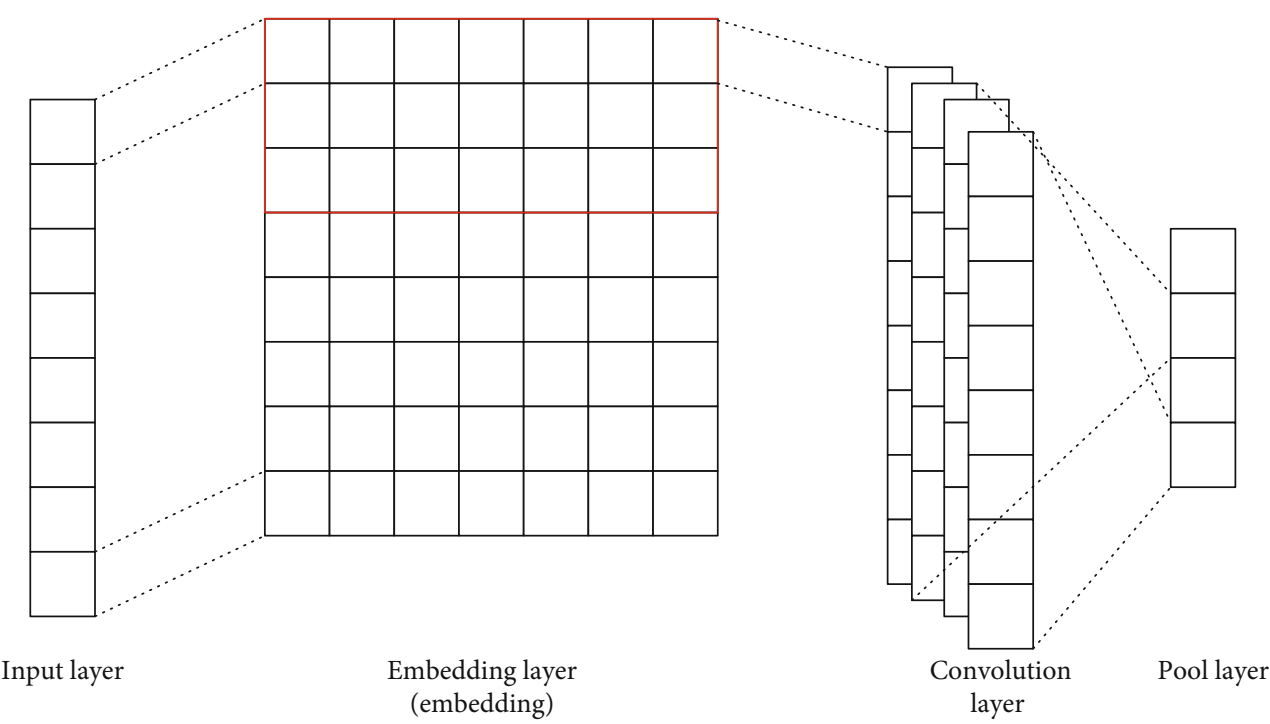

FIGURE 4: Structure diagram of the text convolution neural network.

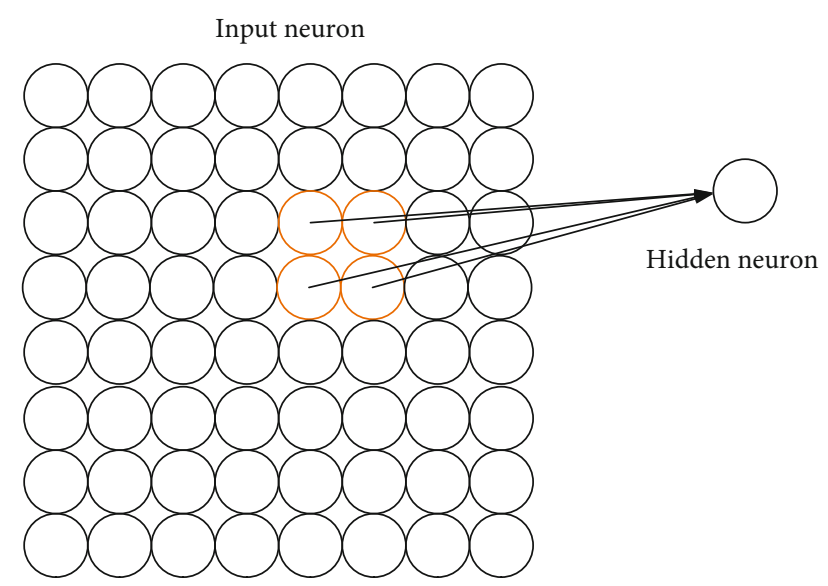

FIGURE 5: Schematic diagram of the local perceptual visual field of the convolution neural network.

After the above convolution operation, the output of the convolution layer can be expressed by

$$
c^{j}=\left[c_{1}^{j}, c_{2}^{j}, \cdots, c_{i}^{j}, \cdots, c_{l}^{j}\right]
$$

In this paper, using the ReLU activation function, the ReLU function can avoid the gradient disappearance problem and avoid falling into the local optimal solution.

(3) Pool Layer. In a convolution neural network, the pool layer follows the convolution layer closely. Its goal is to simplify the output of the convolution layer. In fact, this is a desampling operation. Pooling can reduce the size of data space, thus reducing the number of parameters and calculations.

The pool layer takes the output of the convolution layer as an input and converts the input into a fixed feature map. The most commonly used operation is max-pooling, which takes the maximum value in the local sensory field of a specific size so as to achieve the purpose of dimensionality reduction. The specific operation method can be expressed by

$$
d_{f}=\left[\max \left(c^{1}\right), \max \left(c^{2}\right), \cdots, \max \left(c^{i}\right), \cdots, \max \left(c^{n}\right)\right] .
$$

4.2.4. Regional Characteristics of Entrepreneurial Projects and Domain Characteristics of Entrepreneurial Projects. The regional characteristics and domain characteristics of entrepreneurial projects are coded by one-hot. Taking the region feature processing method as an example, assuming that there are $Q$ region information, the region information of the project is mapped into a $Q$-dimensional vector. Assuming that the region set can be expressed as $a=\left\{a_{1}, a_{2}, \cdots, a_{\mathrm{q}}\right\}$, the region information of the item can be encoded as

$$
[\cdots 0 \cdots \cdots \cdots, \cdots]_{i x q}
$$

Similarly, the domain information of the project can be encoded in this way.

In addition, you can use one-hot encoding to handle lost data. There are a few missing values in the project area information. In this case, the missing value can be incorporated as a code directly into a one-hot encoding. For example, if there are $Q$ regions, adding a "missing" region will become a $Q+1$ region, and the region vector of each item will become a $Q+1$ dimension vector accordingly.

4.2.5. Fusion Layer. In this paper, the horizontal stitching method is adopted directly, and the output of the text convolution neural network is directly stitched into region information, domain information, and label information as the input of the next layer, as shown in

$$
X=\left[X_{1}, X_{2}, \cdots, X_{k}\right]
$$

where $X_{1}, X_{2}, \cdots, X_{k}$ represents the output of various features after corresponding processing. 


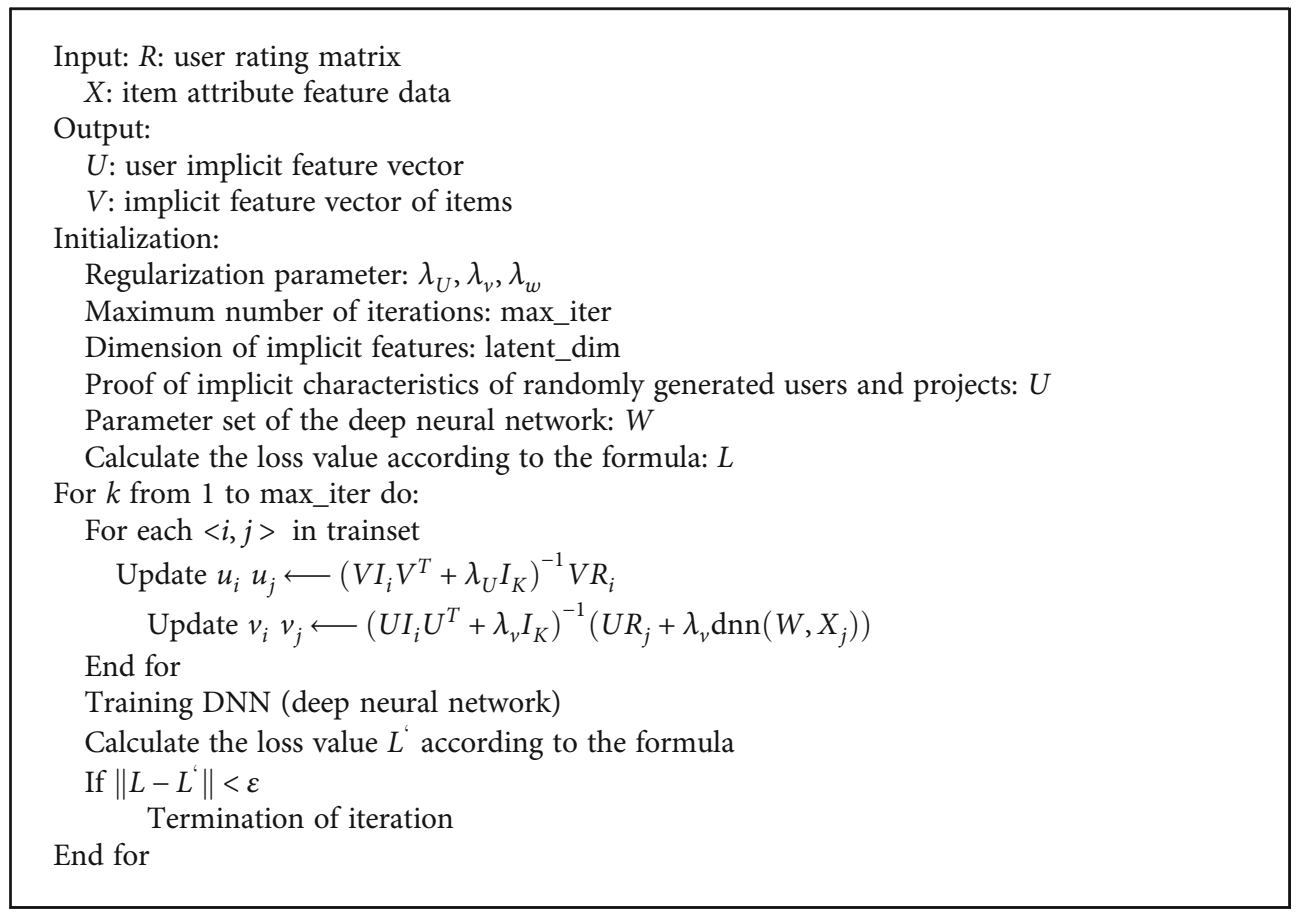

Algorithm 1: Optimization algorithm based on the deep neural network and matrix decomposition.

TABle 5: Schematic diagram of entrepreneurial project data.

\begin{tabular}{lc}
\hline Field & Data \\
\hline Item number & 10005 \\
Project name & VICO Smart Car Box \\
Project area & Automobile traffic/vehicle networking \\
Financing rounds & and hardware \\
Project description & Angel wheel \\
Item label & networking equipment and services \\
Region & Equipment/traffic/vehicle networking \\
Project status & Haiding District, Beijing \\
\hline
\end{tabular}

4.2.6. Full Connection Layer. The combined features need to extract the abstract features of the project through the whole connection layer, and the calculation formula of all connection layers is as shown in

$$
X_{f c}=f\left(W_{f \varepsilon} X+b_{f c}\right)
$$

where $f$ represents the activation function, $W_{f \varepsilon}$ represents the parameter set, and $b_{f c}$ represents the offset set.

4.2.7. Output Layer. The purpose of the output layer is to transform the output of the previous layer into feature vectors contained in items with specific dimensions. The calculation method is shown in

$$
\text { output }=f\left(W W_{f \varepsilon}+b\right)
$$

TABLE 6: Sample table of user information.

\begin{tabular}{lc}
\hline Field & Data \\
\hline $\begin{array}{l}\text { User number } \\
\text { User region }\end{array}$ & $\begin{array}{c}\text { Sichuan Chengdu } \\
\text { User profile }\end{array}$ \\
$\begin{array}{l}\text { Areas of concern } \\
\text { starting a business and setting up overseas } \\
\text { projects, and now I am transforming to } \\
\text { make an investment }\end{array}$ \\
$\begin{array}{l}\text { User's purpose on } \\
\text { the website }\end{array}$ & $\begin{array}{c}\text { Automobile/advertisement/hardware/ } \\
\text { culture/sports/software }\end{array}$ \\
\hline
\end{tabular}

\section{Model Optimization}

To optimize the above model, the optimization objectives are shown in

$$
\begin{gathered}
\max _{U, V, W} P\left(U, V, W \mid R, X, \sigma^{2}, \sigma_{U}^{2} \sigma_{v}^{2}, \sigma_{\mathrm{w}}^{2}\right) \\
=\max _{U, V, W}\left[P\left(R \mid U, V, \sigma^{2}\right) P\left(U \mid \sigma_{U}^{2}\right)\right. \\
\left.\cdot P\left(V \mid W, X, \sigma_{V}^{2}\right) P\left(W \mid \sigma_{W}^{2}\right)\right] .
\end{gathered}
$$

By optimizing formula (22), the optimal $U, V$, and $W$ can be obtained. In this paper, the gradient descent algorithm is adopted, and the first partial derivative of $U$ and $V$ in formula (22) is obtained so that it is equal to $O$, and the gradient descent direction can be obtained, such as

$$
u_{i} \longleftarrow\left(V I_{i} V^{T}+\lambda_{U} I_{K}\right)^{-1} V R_{i},
$$




$$
u_{j} \longleftarrow\left(U I_{j} U^{T}+\lambda_{V} I_{K}\right)^{-1}\left(U R_{j}+\lambda_{v} \operatorname{dnn}\left(W, X_{j}\right)\right),
$$

where $W$ represents the parameter set of the depth neural network, which cannot be solved directly by the above formula, but when $U$ and $V$ are determined, the depth neural network can be established.

The above optimization process can be described by Algorithm 1.

Using the algorithm in the third part, we can get the hidden features $u$ and $v$ of the optimal user and scheme and use $u$ and $v$ to predict the missing score information in the score matrix $R$. The score of user $I$ on item $J$ can be calculated by

$$
r_{i j} \approx \widehat{r_{i j}}=u_{i}^{T} v_{j}
$$

Calculate the user's scores of all items, sort the predicted scores from big to small, and take the first $k$ results as the user's top $k$ recommendation results.

\section{Experiment and Evaluation}

6.1. Data Acquisition and Data Processing. In this paper, a Python crawler is used to capture data from a network information platform. Different information needs to be processed separately, and the processing methods are as follows.

6.1.1. Missing Data Processing. After deleting the data of users with too little action, users with too much data, and entrepreneurial projects, there are 7762 entrepreneurial projects and 7765 users. With the implicit feedback data processing method, the sparsity of 248605 scoring data of users is $99.59 \%$.

6.1.2. Data Processing of Entrepreneurial Projects. Among the entrepreneurial projects, only a few projects have a loss value. Table 5 is an example of entrepreneurial project information.

6.1.3. User Data Processing. The characteristics of the user mainly include the user number, the user area, the user profile, the area of interest of the user, and the purpose on the user's website. Table 6 is an example table representing the characteristic data of the user.

As shown in Table 7, the loss rate of each field of userspecific information can be seen from the table whether the loss rate of user information is large or not. The main reason is that the user's characteristic data is filled in by himself when registering the user, which is unnecessary information. Among them, the missing rate of the user profile is $75.83 \%$, which is too large to be used. The missing rate of user areas, areas of interest, and purposes on the user's websites is about $20 \%$. Because these fields are processed using one-hot encoding, one-hot encoding has the characteristic of being able to handle missing data. Keep these three fields.

6.2. Evaluation Criteria. In this paper, two algorithms of the recommendation system, RMSE and Recall@ $M$, are used to evaluate the experimental results. RMSE represents the
TABLE 7: Missing ratio of each field of user information.

\begin{tabular}{lc}
\hline Field & Deletion rate \\
\hline User number & 0 \\
User region & $23.26 \%$ \\
User profile & $75.83 \%$ \\
Areas of concern to users & $23.38 \%$ \\
User's purpose on the website & $19.80 \%$ \\
\hline
\end{tabular}

mean square error, which is mainly used to evaluate prediction problems. The calculation method is shown in

$$
\mathrm{RMSE}=\sqrt{\frac{1}{|T|} \sum_{i=1}^{|T|}\left(r_{i}-r_{j}\right)^{2} .}
$$

Recall@M is often used in the evaluation of top $N$ recommendations. The calculation method is shown in

$$
\text { Recall@M } M=\frac{\sum_{u}|R(u) \bigcap T(u)|}{\sum_{u} T(u)} .
$$

\subsection{Data Preprocessing}

6.3.1. Text Data Processing. The text introduction information and user description information of business projects need word segmentation.

6.3.2. Word Vector and Pretraining. Use Stanford Segmenter for word segmentation. Then, the wordERC tool provided by Python's Gensim wrapper is used to train the word vector, and finally, about 2.7 million word vectors are obtained.

6.3.3. Feature Extraction. In regional information processing, the data is indeed encoded separately, and finally, there are 44 regions. There are 21 areas of concern to users. There are 9 purposes on the user's website.

6.4. Experimental Results. In this paper, the PMF algorithm and ConvMF algorithm are selected and compared with the recommendation algorithm proposed in this paper. As can be seen from Table 8 , RMSE is $9.83 \%$ higher than that of the PMF algorithm and is $2.97 \%$ higher than that of the CovMF algorithm. As can be seen from Table 8 and Figure 6, the DNN-MF algorithm significantly improves the other two algorithms in the recall indicator.

The recall rate of the DNN-MF algorithm from Figure 6 varies from 50-300, and the DNN-MF has an obvious upward trend, which is higher than the other two algorithms.

6.5. Influence of Adding Different Numbers of Features on Recommendation Results. In this paper, the DNN-MF model is experimented with adding various quantities of item feature information in stages, as shown in Table 9 and Figure 7.

From Table 9, the RMSE index of the DNN-MF1 model is increased by $1.73 \%$ compared with that of the ConvMF model. Compared with the DNN-MF1 model, the RMSE index of the DNN-MF2 model increased by $1.26 \%$. 
TABLE 8: Overall experimental results.

\begin{tabular}{lccccc}
\hline Indicators & RMSE & Recall@50 & Recall@100 & Recall@200 & Recall@300 \\
\hline PMF & 0.6889 & 0.0425 & 0.0736 & 0.1164 & 0.1556 \\
ConvMF & 0.6402 & 0.0869 & 0.1446 & 0.2422 & 0.3181 \\
DNN-MF & 0.6212 & 0.1385 & 0.2044 & 0.2975 & 0.3711 \\
\hline
\end{tabular}

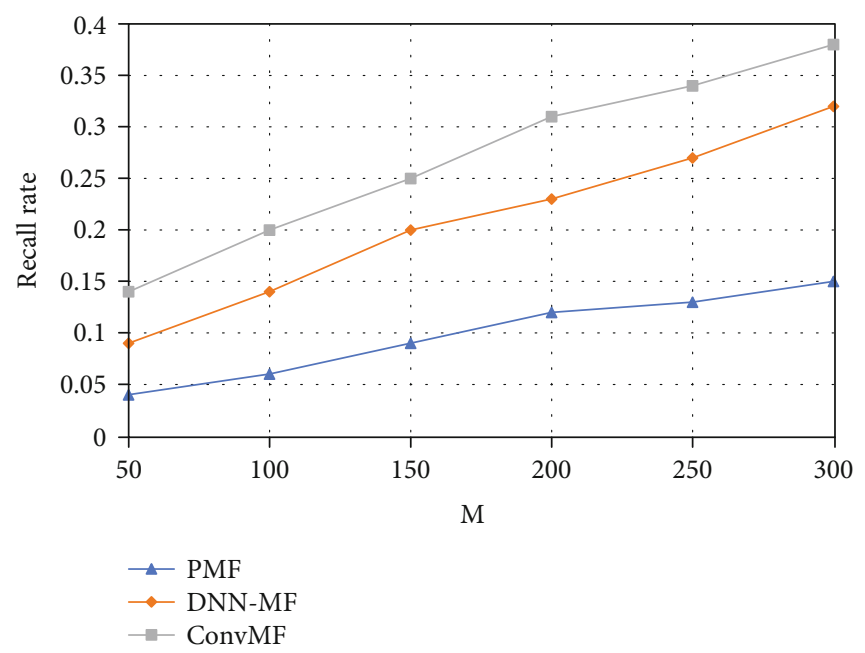

FIgURE 6: Recall rate line chart.

TABLE 9: Influence of adding different numbers of content characteristics on results.

\begin{tabular}{lccccc}
\hline Indicators & RMSE & Recall@50 & Recall@100 & Recall@200 & Recall@300 \\
\hline ConvMF & 0.6402 & 0.0869 & 0.1446 & 0.2421 & 0.3181 \\
DNN-MF1 & 0.6291 & 0.1221 & 0.1861 & 0.2772 & 0.3469 \\
DNN-MF2 & 0.6212 & 0.1385 & 0.2044 & 0.2975 & 0.3710 \\
\hline
\end{tabular}

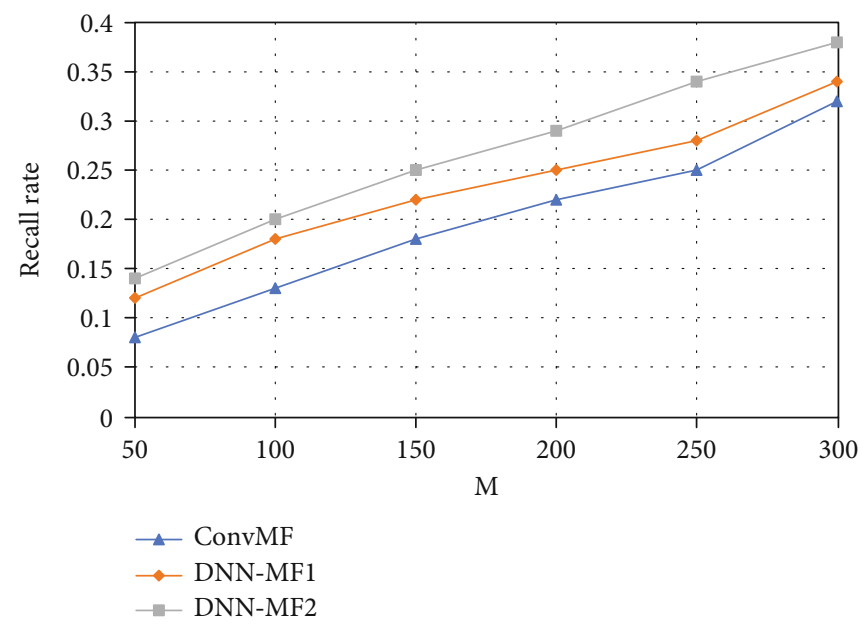

Figure 7: Recall rate line chart.

From the above experimental results, it can be seen that by adding more content features, the recommendation accuracy of the model can be effectively improved. This is mainly because the characteristics of project content can reflect users' interests to a certain extent. For example, for the financing rounds of entrepreneurial projects, some users may pay more attention to the projects in the seed round, while others may pay more attention to the projects after Round A.

6.6. Influence of the Number of Implicit Features and the Length of the Word Vector on Recommendation Results. In 
TABLE 10: Influence of the length of the implicit feature number and word vector number on RMSE.

\begin{tabular}{lccccc}
\hline Implicit feature number & 50 & 100 & 150 & 200 & 250 \\
\hline 100 & 0.6376 & 0.6279 & 0.6252 & 0.6255 & 0.6232 \\
200 & 0.6331 & 0.6265 & 0.6237 & 0.6212 & 0.6264 \\
300 & 0.6313 & 0.6316 & 0.6260 & 0.6246 & 0.6258 \\
\hline
\end{tabular}

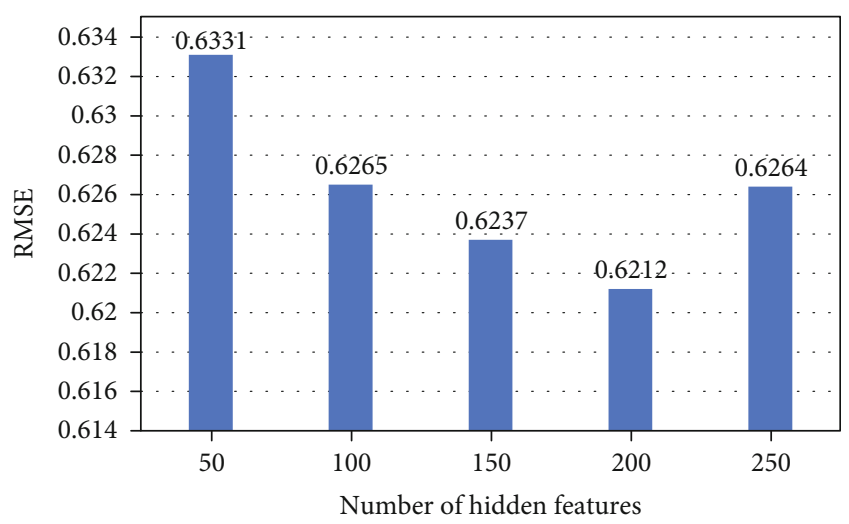

FIGURE 8: Influence of the number of hidden features on RMSE (word vector length: 200).

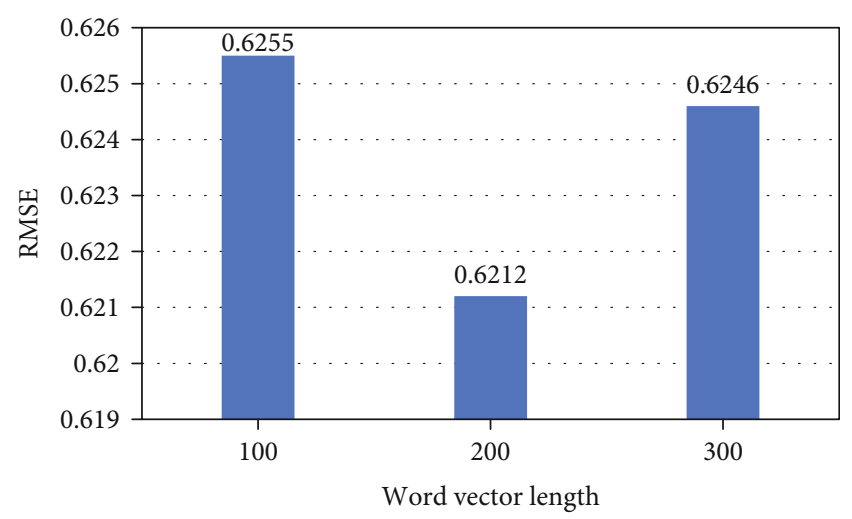

FIGURE 9: Influence of word vector length on recommendation results (number of hidden features: 200).

this paper, the grid search method is adopted. Table 10 and Figures 8 and 9 are experimental results implicitly containing the number of features and the length of the word vector. It can be seen from Table 10 that if the number of features is 200 and the length of the word vector is 200, the RMSE value obtained is the smallest and the recommendation effect is the best.

As you can see from Figure 8, if the word vector dimension is 200, the RMSE value is the smallest. When the number of implicit features increases from 50 to 200, the RMSE value of the algorithm decreases continuously.

Figure 9 is a diagram of the relationship between word vectors of different dimensions and RMSE when the dimension of implicit feature is 200 . As can be seen from the figure, the recommendation effect is best when the length of the word vector is 200 , so the best length of the word vector is 200 .

\section{Conclusion}

Firstly, this paper introduces the basic process of an entrepreneurial project recommendation system based on a deep neural network and matrix decomposition and introduces the internal feedback data processing method and negative sampling method related to this study. Moreover, this paper introduces the principle of the entrepreneurial project recommendation algorithm based on a deep neural network and matrix factorization proposed in this paper. We introduce the principle of probability matrix decomposition and the extraction of hidden features of entrepreneurial projects. Finally, the optimization method of the model and the generation method of recommendation results are introduced.

\section{Data Availability}

The experimental data used to support the findings of this study are available from the corresponding author upon request.

\section{Conflicts of Interest}

The authors declared that they have no conflicts of interest regarding this work.

\section{References}

[1] C. Carrer, G. A. Plonski, C. Carrer, and C. E. L. . Oliveira, "Innovation and entrepreneurship in scientific research," Revista Brasileira de Zootecnia, vol. 39, suppl spe, pp. 17-25, 2010.

[2] X. Hu and Y. Tan, "A study on the reasons of insufficiency of entrepreneurship initiative of Chinese college students," in Communications in Computer and Information Science, vol. 208, pp. 348-352, Springer Berlin Heidelberg, Dalian, China, 2011.

[3] J. A. Cano and A. Tabares, "Determinants of university students' entrepreneurial intention: GUESSS Colombia study," Espacios, vol. 38, no. 45, p. 22, 2017.

[4] W. Ragmoun, "WR A descriptive analysis of entrepreneurial female career success determinants on Saudi Arabia along entrepreneurial process," Archives of Business Research, vol. 7, no. 11, pp. 119-129, 2019.

[5] J. Wichmann, "Grundideen und internationale Rezeption des Modells einer Service University," Zeitschrift für Erziehungswissenschaft, vol. 3, no. 1, pp. 81-96, 2000.

[6] M. C. Pérez-López, M. J. González-López, and L. RodríguezAriza, "Applying the social cognitive model of career selfmanagement to the entrepreneurial career decision: the role of exploratory and coping adaptive behaviours," Journal of Vocational Behavior, vol. 112, pp. 255-269, 2019.

[7] A. L. Merida and V. Rocha, "It's about time: the timing of entrepreneurial experience and the career dynamics of university graduates," Research Policy, vol. 50, no. 1, p. 104135, 2021.

[8] K. D. Cassidy, K. A. Quinn, and G. W. Humphreys, "The influence of ingroup/outgroup categorization on same- and otherrace face processing: the moderating role of inter- versus intraracial context," Journal of Experimental Social Psychology, vol. 47, no. 4, pp. 811-817, 2011. 
[9] K. M. Fems, "Entrepreneurship education in Nigeria tertiary institutions and its impact on students' entrepreneurial career intentions. A study of students at Federal Polytechnic Ekowe," Open Science Journal, vol. 5, no. 4, 2020.

[10] M. Banerjee, S. Biswas, P. Roy, S. Banerjee, S. Kunamaneni, and A. Chinta, "Does career planning drive agri-entrepreneurship intention among university students?," Global Business Review, vol. 4, 2020.

[11] T. Baycan, "Entrepreneurial interest of university students in a multicultural society," in A Broad View of Regional Science, vol. 47, pp. 137-159, 2021.

[12] S. F. F. Binti Shamsudin, A. A. Mamun, N. B. Che Nawi, N. A. B. Md Nasir, and M. N. Bin Zakaria, "Factors affecting entrepreneurial intention among the Malaysian university students," The Journal of Developing Areas, vol. 51, no. 4, pp. 423-431, 2017.

[13] Z. K. Zhang, T. Zhou, and Y. C. Zhang, "Personalized recommendation via integrated diffusion on user-item-tag tripartite graphs," Physica A Statistical Mechanics \& Its Applications, vol. 389, no. 1, pp. 179-186, 2010.

[14] Y. Guo, M. Wang, and X. Li, "Application of an improved Apriori algorithm in a mobile e-commerce recommendation system," International journal of entrepreneurial behaviour \& research, vol. 23, no. 2, pp. 287-303, 2017.

[15] Y. Wang, H. Y. Ou, and J. M. Zhang, "Design and implementation of e-commerce recommendation system based on ontology technology," Advanced Materials Research, vol. 978, pp. 244-247, 2014.

[16] J. Huang, B. Jiang, J. Pei, J. Chen, and Y. Tang, "Skyline distance: a measure of multidimensional competence," Knowledge and Information Systems, vol. 34, no. 2, pp. 373-396, 2013.

[17] L. Liu, J. Xu, S. S. Liao, and H. Chen, “A real-time personalized route recommendation system for self-drive tourists based on vehicle to vehicle communication," Expert Systems with Applications, vol. 41, no. 7, pp. 3409-3417, 2014.

[18] O. Khalid, M. Khan, S. U. Khan, and A. Y. Zomaya, “OmniSuggest: a ubiquitous cloud-based context-aware recommendation system for mobile social networks," IEEE Transactions on Services Computing, vol. 7, no. 3, pp. 401-414, 2014.

[19] G. Chen and S. Li, "Research on location fusion of spatial geological disaster based on fuzzy SVM," Computer Communications, vol. 153, pp. 538-544, 2020.

[20] S. Li, Y. Zhang, M. Xie, and H. Sun, "Belief reasoning recommendation -mashing up web information fusion and FOAF," Journal of Computers, vol. 5, no. 12, pp. 1885-1892, 2010. 